\title{
Policies make coherent care pathways a personal responsibility for clinicians: A discourse analysis of policy documents about coordinators in hospitals
}

Audhild Høyem, Deede Gammon, Gro Rosvold Berntsen and Aslak Steinsbekk

\section{List of analysed documents}

Accessed 6 July 2018

\begin{tabular}{|c|c|}
\hline $\begin{array}{l}\text { Document number, document title and which } \\
\text { parts of the documents are analysed }\end{array}$ & URL \\
\hline $\begin{array}{l}\text { 1. Specialized Health Services Act [12]. §§ 2-2, } \\
2-5 a, b \text { and c }\end{array}$ & https://lovdata.no \\
\hline $\begin{array}{l}\text { 2. Regulations to the Specialized Health Services } \\
\text { Act and the Health and Care Services Act } \\
\text { concerning rehabilitation, individual plan and } \\
\text { patient care coordinator [28] }\end{array}$ & https://lovdata.no \\
\hline $\begin{array}{l}\text { 3. Directive to the Specialized Health Services } \\
\text { Act [26]. P. 23-27 }\end{array}$ & $\begin{array}{l}\text { https://helsedirektoratet.no/publikasjoner/sp } \\
\text { esialisthelsetjenesteloven-med-kommentarer }\end{array}$ \\
\hline $\begin{array}{l}\text { 4. Law proposition to the Parliament, } \\
\text { Prop. } 125 \text { L. Amendments to the Specialized } \\
\text { Health Care Act. [25] Chapters 1-8, p. 5-38 } \\
\text { and 10, p. } 43-46\end{array}$ & $\begin{array}{l}\text { https://www.regjeringen.no/no/dokumenter/ } \\
\text { prop.-125-I-20142015/id2412753/ }\end{array}$ \\
\hline $\begin{array}{l}\text { 5. Guidelines for patient care coordinator [29]. } \\
\text { Chapter } 13, \text { p. } 82-93\end{array}$ & $\begin{array}{l}\underline{\text { https://helsedirektoratet.no/Retningslinjer/Re }} \\
\underline{\text { habilitering,\%20habilitering,\%20individuell\%2 }} \\
\underline{\text { Oplan\%20og\%20koordinator.pdf. }}\end{array}$ \\
\hline $\begin{array}{l}\text { 6. Guidelines for contact physician [30]. } \\
\text { Chapters } 1-8, \text { p. } 1-33\end{array}$ & $\begin{array}{l}\text { https://helsedirektoratet.no/retningslinjer/vei } \\
\text { leder-for-kontaktlege-i- } \\
\text { spesialisthelsetienesten. }\end{array}$ \\
\hline $\begin{array}{l}\text { 7. The Coordination Reform. Proper treatment - } \\
\text { at the right place and right time. Report No. } \\
47 \text { (2008-2009) to the Storting. [11]. } \\
\text { Chapters 1-5, p.11-53 and 10, p. 111-114 }\end{array}$ & $\begin{array}{l}\text { https://www.regjeringen.no/no/dokumenter/ } \\
\text { stmeld-nr-47-2008-2009-/id567201/ }\end{array}$ \\
\hline $\begin{array}{l}\text { 8. NOU 2005: 3. From piecemeal to whole - an } \\
\text { integrated health service [31]. Chapters 1, } 2 \text {, } \\
\text { p. } 11-21,4, \text { p. } 40-48,6 \text { and } 7 \text {, p. } 67-87\end{array}$ & $\begin{array}{l}\text { https://www.regjeringen.no/no/dokumenter/ } \\
\text { nou-2005-03/id152579/. }\end{array}$ \\
\hline $\begin{array}{l}\text { 9. NOU 1997: 2. The patient first! Leadership } \\
\text { and organization in hospitals [32]. Chapters 2, } \\
\text { p.15-16 and 8, p.92-108 }\end{array}$ & $\begin{array}{l}\text { https://www.regjeringen.no/no/dokumenter/ } \\
\text { nou-1997-2/id140689/sec1 }\end{array}$ \\
\hline $\begin{array}{l}\text { 10. Meld.St.11 (2015-2016) National health- and } \\
\text { hospital plan 2016-2019. [33]. Chapter 7.3, p. } \\
\text { 57-58 }\end{array}$ & $\begin{array}{l}\text { https://www.regjeringen.no/no/dokumenter/ } \\
\text { meld.-st.-11-20152016/id2462047/ }\end{array}$ \\
\hline
\end{tabular}

